\title{
A Fibreless Multiwavelength NIRS System for Imaging Localised Changes in Cerebral Oxidised Cytochrome C Oxidase
}

\author{
D. Highton, D. Chitnis, S. Brigadoi, P. Phan, \\ I. Tachtsidis, R. Cooper, N. Everdell, J. Hebden, \\ M. Smith, and C. E. Elwell
}

\begin{abstract}
Measurement of the oxidation state of cytochrome c oxidase (oxCCO) can inform directly on neuronal metabolism. Conventionally this has been measured in vivo using benchtop broadband near infrared spectroscopy (NIRS) systems. Spatially resolved measures of oxCCO have recently been made possible using a multichannel fibre-based broadband NIRS system. We describe the use of a fibreless multiwavelength NIRS system using light emitting diodes (LED) designed specifically to image localised changes in oxCCO and hence neuronal metabolism. A
\end{abstract}

The original version of this chapter was revised. The correction to this chapter is available at https://doi.org/10.1007/978-3-319-91287-5_70

\section{Highton}

Neurocritical Care Unit, National Hospital for Neurology and Neurosurgery, London, UK

University of Queensland, Brisbane, Australia

D. Chitnis · P. Phan · I. Tachtsidis · R. Cooper

N. Everdell · J. Hebden · C. E. Elwell $(\bowtie)$

Department of Medical Physics and Biomedical

Engineering, UCL, London, UK

e-mail: c.elwell@ucl.ac.uk

\section{S. Brigadoi}

Department of Medical Physics and Biomedical Engineering, UCL, London, UK

Department of Developmental and Social Psychology, University of Padova, Padua, Italy fibreless system consisting of four modules, each containing two LED sources and four photodiode detectors, was developed. Each LED source contained eight LED dies (780, $811,818,842,850,882,891$ and $901 \mathrm{~nm}$ ) assembled in an area of $1.5 \times 1.5 \mathrm{~mm}$. A wellestablished hyperoxia protocol was used to evaluate the oxCCO spatially resolved measurement capabilities of the system and, subsequently, its imaging capabilities were tested using a functional activation paradigm. A multi-spectral image reconstruction approach was used to provide images of $\Delta\left[\mathrm{HbO}_{2}\right]$, $\Delta[\mathrm{HHb}]$ and $\Delta[\mathrm{oxCCO}]$ from the multidistance, multi-channel optical datasets. This novel fibreless multiwavelength NIRS system allows imaging of localised changes in oxCCO in the human brain, and has potential for development as an inexpensive, wearable, continuous monitor of cerebral energetics in a range of experimental and clinical scenarios. 


\section{Introduction}

Near-infrared spectroscopy (NIRS) provides the capability to non-invasively characterise cerebral haemodynamics and metabolism by using the attenuation of near infrared light to derive concentration changes in oxyhaemoglobin $\Delta\left[\mathrm{HbO}_{2}\right]$, deoxyhaemoglobin $\Delta[\mathrm{HHb}]$ and the oxidation state of cytochrome $\mathrm{c}$ oxidase $\Delta$ [oxCCO] [1]. To date NIRS studies of functional brain activation have monitored changes in $\left[\mathrm{HbO}_{2}\right]$ and $[\mathrm{HHb}]$ to measure the haemodynamic response resulting from neuronal activation. Detailed $\left[\mathrm{HbO}_{2}\right] /[\mathrm{HHb}]$ maps of localised functional responses have been reported in several studies using diffuse optical imaging [2] and, more recently, with wearable or fibreless systems [3]. Given the role of cytochrome c oxidase as the terminal electron acceptor in the mitochondrial electron transport chain, the NIRS-derived oxCCO measure of the oxidation status of this enzyme is well placed to provide a direct marker of cellular oxygen metabolism. Conventional measures of $\triangle \mathrm{oxCCO}$ have been achieved in vivo using benchtop broadband near infrared spectroscopy (NIRS) systems [4], but a recently [5] described multichannel fibrebased broadband NIRS system is capable of delivering spatially resolved measures of [oxCCO]. Here we present, for the first time, a fibreless multiwavelength NIRS system using light emitting diodes (LED) designed specifically to image localised changes in [oxCCO] and hence neuronal metabolism. We used a well-established hyperoxia protocol to demonstrate the CCO measurement capabilities of the system, and then tested its imaging capabilities using a functional activation paradigm.

\section{Methods}

The fibreless system consists of four modules each containing two multiwavelength light emitting diodes (LED) sources (OSA_Opt, Germany) and four photodiode detectors (Hamamatsu Photonics, Japan). Each source contains eight LED dies (780, 811, 818, 842, 850, 882, 891 and $901 \mathrm{~nm}$ ) assembled in an area of $1.5 \times 1.5 \mathrm{~mm}$ based on the findings of [6]. The optoelectronics for the modular system are described in detail in [7] and the production of the multiwavelength sources in [8]. Figure 1 is a schematic of a single module incorporating two multiwavelength light sources and four detectors.

Following ethical approval and volunteer consent, the system was used to collect data during two studies, one involving a hyperoxia challenge in ten healthy volunteers and the other a hemifield stimulation of the visual cortex in a single volunteer.

\subsection{Hyperoxia Studies}

The array was positioned over Fp1 (10-20 electrode placement system). The experimental protocol involved $2 \mathrm{~min}$ of baseline normoxia, $5 \mathrm{~min}$ of hyperoxia followed by $5 \mathrm{~min}$ of normoxia. NIRS data were collected using all eight wavelengths from one light source on each of two modules and a total of eight detectors, resulting in a source-detector separation of $3.5 \mathrm{~cm}$. Data were averaged across channels and across subjects.

\subsection{Hemifield Stimulation Studies}

The array was centred over the primary visual cortex (O1 position). A $2 \mathrm{~Hz}$ inverting hemifield checkerboard stimulus of $20 \mathrm{~s}$ duration on each hemifield (20 s on left and $20 \mathrm{~s}$ on right) was repeated 15 times and averaged. Data were collected from all source and detector positions on all four modules but, due to the repetition rate of the switching between wavelengths, only four wavelengths $(780,811,842,891)$ were used at each source as defined by [6]. A multi-spectral image reconstruction approach was used to reconstruct images of $\Delta\left[\mathrm{HbO}_{2}\right], \Delta[\mathrm{HHb}]$ and $\Delta[$ oxCCO] from the multi-distance, multi-channel optical datasets. TOAST++ software was used to run the forward model on the registered adult volumetric mesh. The LSQR method was employed to solve the inverse problem with first-order Tikhonov regularization. The volumetric images were then projected on the cortical surface mesh [9]. 


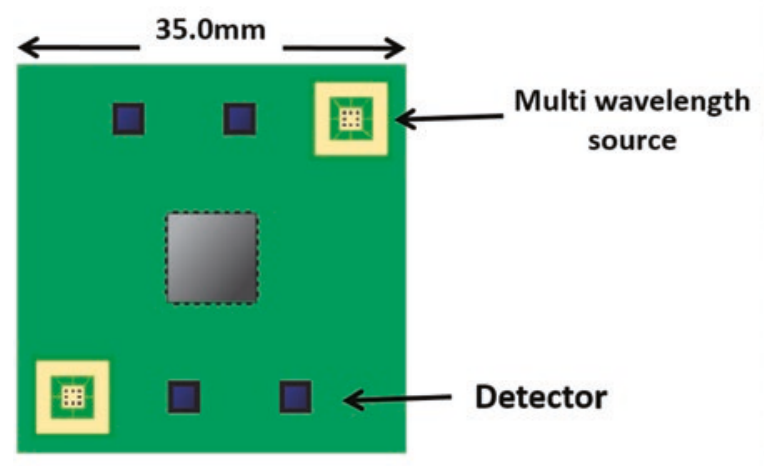

(a)

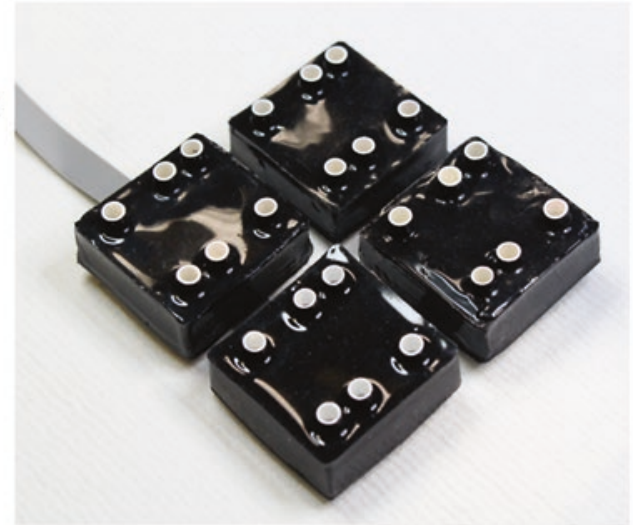

(b)

Fig. 1 (a) Schematic of single module containing two multi-wavelength sources and four detectors, (b) photograph of the four-module system

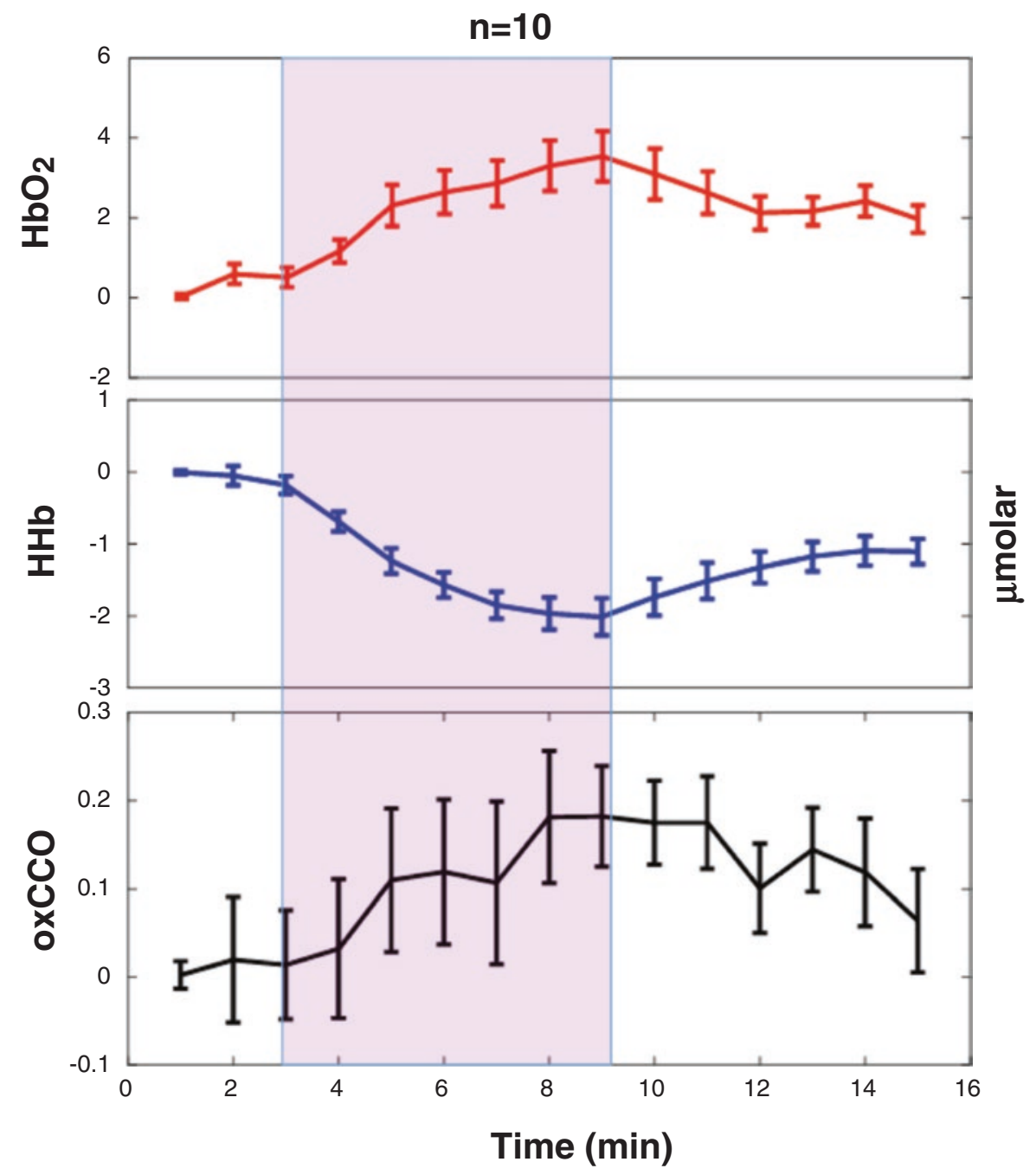

Fig. 2 Mean and standard deviation of $\Delta\left[\mathrm{HbO}_{2}\right], \Delta[\mathrm{HHb}]$ and $\Delta[\mathrm{oxCCO}]$ from ten subjects during hyperoxia challenge 
a

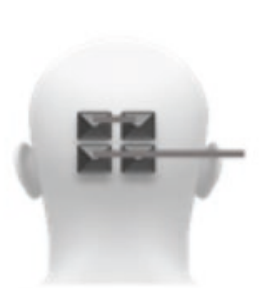

C $\quad \Delta \mathrm{HbO}_{2}$
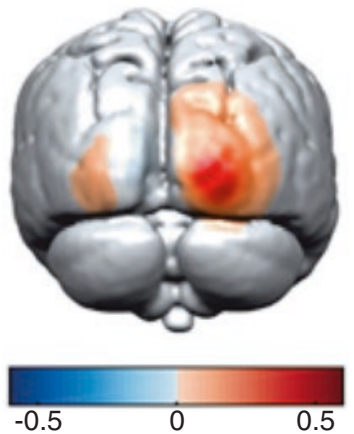

b

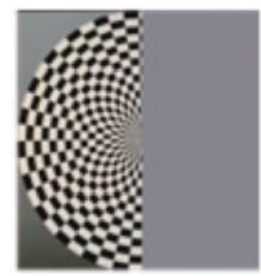

$\Delta \mathrm{HHb}$
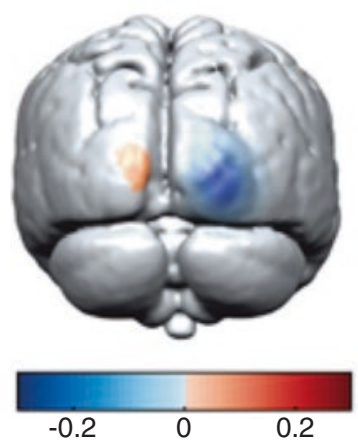

$\triangle \mathrm{OxCCO}$
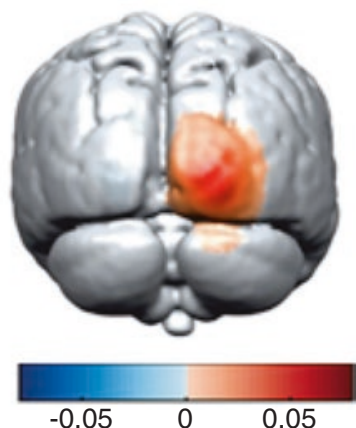

Fig. 3 (a) Four-module array centred over the occipital cortex, (b) checkerboard stimulus activating left visual field, (c) images of $\Delta\left[\mathrm{HbO}_{2}\right], \Delta[\mathrm{HHb}]$ and $\Delta[\mathrm{oxCCO}]$ showing activation on right visual cortex (colour scale in $\mu$ molar)

\section{Results}

Figure 2 shows the data collected during the hyperoxia studies averaged across channels and across subjects; the measurement changes are consistent with previous studies using standard broadband NIRS systems [10]. Figure 3 shows the $\Delta\left[\mathrm{HbO}_{2}\right], \Delta[\mathrm{HHb}]$ and $\Delta[\mathrm{oxCCO}]$ images reconstructed from the left hemifield stimulation study.

\section{Discussion}

We have demonstrated the development and testing of a novel fibreless multiwavelength NIRS system with specific capability to measure localised changes in oxCCO. The hyperoxia study confirms the eight wavelength capability of the system over two modules without spatial discrimination, and the hemifield stimulation the capability of a four-module system to deliver depth-resolved images of regional changes in oxCCO. These tests demonstrate a flexible approach to multi-wavelength, multi-channel NIRS measures. Although further work is required to increase functionality and implemen- tation in a clinical setting, this approach has potential to deliver an inexpensive, wearable, continuous monitor of cerebral energetics in a range of experimental and clinical scenarios.

Monitoring and management of patients with acute brain injury (ABI) remain key drivers for the development of NIRS oxCCO systems [11]. Derangements in cerebral metabolism are implicated in the pathophysiology of both secondary brain injury and long-term neurocognitive outcome. Spatially resolved measures of oxCCO may have potential to clarify pathways of injury after ABI, assess the impact of therapies on both injured and non-injured brain regions and, ultimately, guide interventions designed to prevent or minimise secondary brain injury. The advent of fibreless, and therefore wearable, systems capable of making these measures will allow brain monitoring in the post- acute/rehabilitation phase and has potential to elucidate the evolution of long-term neurocognitive damage.

Fibreless systems are also likely to play an increasingly important role in neurodevelopment studies given the additional flexibility they provide for imaging brain function in naturalistic environments, especially for typically and atypically developing young infants and toddlers. 
Recent studies have demonstrated the first measurement of oxCCO changes during functional activation in the infant brain using a single source detector system [12], and further work is already underway to deliver spatially resolved measures of oxCCO to investigate links between mitochondrial and metabolic dysfunction in infants at high risk of autism $[13,14]$.

In addition, as the use of NIRS extends to global health projects (see www.globalfnirs.org) the advent of wearable, relatively low cost, flexible, easy to implement systems will further enhance the applicability of NIRS for studies in resource poor settings [15].

Acknowledgments This work has been funded by the EPSRC (EP/K020315/1). IT is supported by the Wellcome Trust (088429/Z/09/Z and 104580/Z/14/Z). RC is supported by EP/N025946/1. MS was part funded by the UCLH/UCL National Institute for Health Research Biomedical Research Centre.

\section{References}

1. Elwell CE, Cooper CE (2011) Making light work: illuminating the future of biomedical optics. Phil Trans R Soc 369:4355-4357

2. Eggebretcht A, Ferradal S, Robichaux-Viehoever A et al (2014) Mapping distributed brain function and networks with diffuse optical tomography. Nat Photonics 8:448-454

3. Piper SK, Krueger A, Koch SP et al (2014) A wearable multi-channel fNIRS system for brain imaging in freely moving subjects. NeuroImage 85:64-71

4. Bale G, Elwell CE, Tachtsidis I (2016) From Jöbsis to the present day: a review of clinical near-infrared spectroscopy measurements of cerebral cytochromec-oxidase. J Biomed Opt 9:091307
5. Phan P, Highton D, Lai J et al (2016) Multi-channel multi-distance broadband near infrared spectroscopy system to measure the spatial response of cellular oxygen metabolism and tissue oxygenation. Biomed Opt Express 7(11):4424-4440

6. Arifler D, Zhu T, Madaan S et al (2015) Optimal wavelength combinations for near-infrared spectroscopic monitoring of changes in brain tissue hemoglobin and cytochrome c oxidase concentrations. Biomed Opt Express 6(3):933-947

7. Chitnis D, Cooper R, Dempsey L et al (2016) Functional imaging of the human brain using modular, fibreless, high density diffuse optical tomography. Biomed Opt Express 7(10):4275-4288

8. Chitnis D, Airantzis D, Highton D et al (2016) Towards a wearable near infrared spectroscopic probe for monitoring concentrations of multiple chromophores in biological tissue in vivo. Rev Sci Instrum 87(6):065112

9. Brigadoi S, Phan P, Highton D et al (2017) Image reconstruction of oxidized cerebral cytochrome $\mathrm{C}$ oxidase changes from broadband near infrared spectroscopy data. Neurophotonics 4(2):021105

10. Tachtsidis I, Tisdall M, Leung $\mathrm{T}$ et al (2009) Relationship between brain tissue haemodynamics, oxygenation and metabolism in the healthy human adult brain during hyperoxia and hypercapnea. Adv Exp Med Biol 645:315-320

11. Ghosh A, Highton D, Kolyva C et al (2017) Hyperoxia results in increased aerobic metabolism following acute brain injury. J Cereb Blood Flow Metab 37(8):2910-2920

12. Siddiqui M, Lloyd-Fox S, Kaynezhad P et al (2017) Non invasive measurement of a metabolic marker of infant brain function. Sci Rep 7(1):1330

13. Siddiqui M, Elwell CE, Johnson MJ (2016) Mitochondrial dysfunction in autism spectrum disorders. Autism Open Access 6(5):1000190

14. Lloyd-Fox S, Blasi A, Elwell CE et al (2013) Reduced neural sensitivity to social stimuli in infants at risk for autism. Proc Biol Soc 280:20123026

15. Lloyd-Fox S, Papademetrious M, Darboe $M$ et al (2014) Functional near infrared spectroscopy (fNIRS) to assess cognitive function in infants in rural. Afr Sci Rep 4:474

Open Access This chapter is licensed under the terms of the Creative Commons Attribution 4.0 International License (http://creativecommons.org/licenses/by/4.0/), which permits use, sharing, adaptation, distribution and reproduction in any medium or format, as long as you give appropriate credit to the original author(s) and the source, provide a link to the Creative Commons license and indicate if changes were made.

The images or other third party material in this chapter are included in the chapter's Creative Commons license, unless indicated otherwise in a credit line to the material. If material is not included in the chapter's Creative Commons license and your intended use is not permitted by statutory regulation or exceeds the permitted use, you will need to obtain permission directly from the copyright holder.

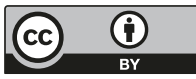

